Balbino, CM, Silvino, ZR, Joaquim, FL, Souza, CJ, Costa, APBF, Moura, LLF. (2020). Gel to decrease adhesion between protective gloves and tape. Research, Society and Development, 9(7): 1-16, e173974042.

\title{
Gel para diminuição da adesão entre luvas protetoras e esparadrapo
}

Gel to decrease adhesion between protective gloves and tape

\section{Gel para disminuir la adhesión entre guantes protectores y cinta}

Recebido: 28/04/2020 | Revisado: 28/04/2020 | Aceito: 30/04/2020 | Publicado: 03/05/2020

\section{Carlos Marcelo Balbino}

ORCID: http://orcid.org/0000-0003-0763-3620

Universidade Federal Fluminense, Brasil

E-mail: carlosmbalbino@hotmail.com

Zenith Rosa Silvino

ORCID: http://orcid.org/0000-0002-2848-9747

Universidade Federal Fluminense, Brasil E-mail: zenithrosa@id.uff.br

Fabiana Lopes Joaquim

ORCID: http://orcid.org/0000-0003-1344-2740

Universidade Federal Fluminense, Brasil

E-mail: fabykim_enf@yahoo.com.br

Cláudio José de Souza

ORCID: http://orcid.org/0000-0001-7866-039X

Universidade Federal Fluminense, Brasil

E-mail: claudioenfo@gmail.com

Ana Paula Bergmann Furtado Costa

ORCID: http://orcid.org/0000-0002-0219-5046

Centro Universitário de Valença, Brasil

E-mail: anabergmann2010@ hotmail.com

Lívia Luiz de Fátima Moura

ORCID: http://orcid.org/0000-0002-6602-4481

Centro Universitário de Valença, Brasil

E-mail: liviadefatimamoura.23@gmail.com 


\title{
Resumo
}

Este estudo teve como objetivo avaliar a usabilidade do gel para utilização em luvas para melhor manuseio com esparadrapos. Para tal foi realizado um estudo exploratório-descritivo com abordagem qualitativa, realizado no Hospital Escola, com enfermeiros assistenciais que receberam gel para a realização de suas atividades laborais em procedimentos que envolvessem o uso de luvas e esparadrapos. Diante da análise foi possível refletir que se evidenciaram como categorias temáticas as dificuldades vivenciadas durante a utilização de luvas em procedimentos que contenham utilização de esparadrapos; rompimento das luvas e o desperdício de material; dificuldades e comprometimento da assistência relacionada à associação luvas-esparadrapo; os benefícios do gel durante as ações assistenciais. Ante ao exposto foi possível concluir que, o uso do gel como tecnologia para evitar a aderência entre as luvas e o esparadrapo em decorrência da cola é positivo e apresenta usabilidade que tende a favorecer a assistência não apenas dos profissionais de enfermagem, mas dos diversos profissionais de saúde.

Palavras-chave: Luvas protetoras; Fita cirúrgica; Saúde do trabalhador; Equipamentos e Provisões; Pesquisa em Administração de Enfermagem.

\begin{abstract}
This study aimed to evaluate the usability of the gel for use in gloves for better handling with adhesive tapes. To this end, an exploratory-descriptive study with a qualitative approach was carried out, carried out at the Hospital School, with nursing assistants who received gel for carrying out their work activities in procedures involving the use of gloves and tape. In view of the analysis, it was possible to reflect that the difficulties experienced during the use of gloves in procedures that contain the use of adhesive tape became evident as thematic categories; breaking the gloves and wasting material; difficulties and impairment of care related to the gloves-adhesive association; the benefits of the gel during assistance actions. Given the above, it was possible to conclude that the use of gel as a technology to prevent adhesion between the gloves and the adhesive tape due to the glue is positive and has usability that tends to favor the assistance not only of nursing professionals, but of the various professionals of health.
\end{abstract}

Keywords: Protective gloves; Surgical tape; Worker's health; Equipment and Supplies; Nursing Administration Research. 


\section{Resumen}

Este estudio tuvo como objetivo evaluar la usabilidad del gel para su uso en guantes para un mejor manejo con cinta. Para ello, se realizó un estudio exploratorio descriptivo con enfoque cualitativo, realizado en la Escuela Hospitalaria, con auxiliares de enfermería que recibieron gel para realizar sus actividades laborales en procedimientos que implican el uso de guantes y cinta adhesiva. En vista del análisis, fue posible reflejar que las dificultades experimentadas durante el uso de guantes en procedimientos que contienen el uso de cinta adhesiva se hicieron evidentes como categorías temáticas; romper los guantes y desperdiciar material; dificultades y deterioro de la atención relacionada con la asociación guantes-adhesivo; Los beneficios del gel durante las acciones de asistencia. Dado lo anterior, fue posible concluir que el uso del gel como tecnología para evitar la adhesión entre los guantes y la cinta adhesiva debido al pegamento es positivo y tiene una usabilidad que tiende a favorecer la asistencia no solo de los profesionales de enfermería, sino de los diversos profesionales de salud.

Palabras clave: Guantes protectores; Cinta quirúrgica; Salud ocupacional; Equipo y Suministros; Investigación de la Administración de Enfermería.

\section{Introdução}

Os equipamentos de proteção individual (EPI) são definidos de acordo com a Norma regulamentadora 6 (NR 6) como dispositivos ou produtos de uso pessoal, usado pelo trabalhador com a finalidade de proteger-se de riscos que ameacem a sua segurança ou saúde no desenvolvimento do trabalho deste modo, há EPIs destinados a proteção da cabeça, olhos e face, auditiva, respiratória, tronco, membros superiores, membros inferiores, corpo inteiro e contra quedas com diferença de nível (Brasil, 2006). Logo, frente aos inúmeros tipos de EPIs, a adoção deste será de acordo com o risco e função que o funcionário esteja desempenhando.

Deste modo, quando nos referimos aos profissionais da área da saúde, nos deparamos com trabalhadores expostos a agentes químicos, físicos, biológicos e ergonômicos (Sulzbacher \& Fontana, 2018), devendo a utilização dos EPIS não ser negligenciada, por ser esta a forma de proteção para referidos agentes agressores presentes nos ambientes hospitalares e ambulatoriais; bem como os agentes psicossociais (Sulzbacher \& Fontana, 2018) por vezes negligenciados.

Dentre os equipamentos de proteção individual mais utilizados pelos profissionais de saúde evidenciamos o uso das luvas (Vieira et.al., 2015) (Barbosa, et. al., 2016), mas embora 
este seja o EPI mais utilizado, sua adoção é por vezes negligenciada por dificultar a execução de técnicas ou escolha de situações em que se acredita existir uma exposição seletiva (Vieira et.al., 2015).

Ao pensarmos nas dificuldades que os profissionais de saúde apresentam para executar os procedimentos com luvas podemos intensificar esta problemática ao uso de esparadrapos, visto que estes apresentam uma face porosa e outra face com cola adesiva que gruda excessivamente no látex causando desconforto aos profissionais, danos às luvas e outros eventos adversos que podem comprometer a assistência e a saúde do profissional. Destarte, evidencia-se que as adversidades com o uso da luva podem fazer com que profissionais negligenciem o uso rotineiro deste artefato (Barros, 2016), principalmente se houver dificuldades atreladas ao uso durante a assistência como o rompimento do EPI.

$\mathrm{O}$ uso dos EPIs exigem treinamentos para que o uso seja efetivo a finalidade que se destina e quando nos referimos às luvas não é diferente. O referido artefato conforme preconiza a Agencia Nacional de Vigilância Sanitária (ANVISA) (2008) RDC nº 05 /2008, apresenta utilização única; deve ser colocado antes da execução de procedimentos; trocado nas diferentes atividades/cuidados com o mesmo paciente, bem como entre os cuidados com diferentes pacientes e descartado imediatamente após o termino da atividade assistencial, visto que o uso em situações não recomendadas representa desperdício de recursos sem, necessariamente, levar à redução de transmissão cruzada de microrganismos, bem como também pode acarretar na redução da higienização das mãos (Brasil, 2018).

Neste ínterim em que discutimos a importância do uso das luvas atrelada a não adesão dos profissionais discutimos também o papel do enfermeiro no gerenciamento dos custos e do cuidado nas unidades de saúde, sendo este um desafio, principalmente se o custo com a utilização do EPI acima mencionado tiver suas compras elevadas por conta da associação luvas e esparadrapo bem como se o cuidado for comprometido em decorrência da insegurança advinda do risco de romper-se a luva ao aderir-se ao esparadrapo, expondo o profissional ao risco de contaminação, e a insatisfação, pelo fato de, muitas vezes, por conta desta adesão, perder-se acessos venosos nos casos de punções difíceis e de urgência, onde um acesso venoso pode determinar a preservação de uma vida, por tratar-se de via de acesso para a administração de soluções, medicamentos, sangue e derivados (Nascimento, 2005).

Deste modo, torna-se necessário avaliar a usabilidade de um gel que impeça a aderência da luva ao esparadrapo assegurando melhor usabilidade pelos profissionais de saúde durante a assistência, visto ser a usabilidade conceituada como condição de ser mais fácil que a utilização, indo além da simples facilidade de usar, referindo-se a um atributo de 
(CC BY 4.0) | ISSN 2525-3409 | DOI: http://dx.doi.org/10.33448/rsd-v9i7.4042

qualidade que se relaciona à facilidade do uso de algo (Meneses, Sobreira \& Rosemberg, 2016), sendo sistema, produto ou serviço por usuários específicos para atingir objetivos específicos com eficácia, eficiência e satisfação em um contexto específico de uso (ISO, 2018).

Diante do exposto, este artigo foi desenvolvido pautado nas seguintes perguntas de investigação: Quais as dificuldades vivenciadas pelos enfermeiros durante o manejo de esparadrapo com luvas de procedimento? Com a utilização do produto, que dificuldades foram sanadas durante o uso de luvas nos procedimentos com esparadrapos?

Este estudo teve como objetivo avaliar a usabilidade do gel para utilização em luvas para melhor manuseio com esparadrapos.

\section{Metodologia}

O estudo atendeu à Resolução ${ }^{\circ}$ 466, de 12 de dezembro de 2012, do Conselho Nacional de Saúde/Ministério da Saúde, sendo submetido à Plataforma Brasil e recebendo parecer aprovação para o seu desenvolvimento.

Trata-se de um estudo exploratório-descritivo com abordagem qualitativa.

Utilizou-se como hipótese para o estudo, o gel condutor utilizado na realização de eletrocardiogramas pode ajudar a evitar aderência entre as luvas e esparadrapos durante a assistência de enfermagem.

O estudo primário, de onde emergiu os resultados presentes neste artigo, foi desenvolvido em um Hospital Escola (HE) numa cidade do interior Sul do Estado do Rio de janeiro, por ser a única instituição médica no município que trabalha com pesquisa e ensino, desta forma, sendo um centro formador de conhecimento científico contribuído para o desenvolvimento de conceitos e práticas médicas e de saúde. A coleta de dados ocorreu de junho a julho de 2017.

O estudo contou com 34 enfermeiros que realizam atividades laborais assistenciais nas diversas unidades de atendimento no HE, totalizando $100 \%$ dos enfermeiros da instituição. Os critérios de inclusão adotados foram: aceitar realizar procedimentos com luvas em procedimentos que envolvam o uso de esparadrapos e os de exclusão: enfermeiros que utilizam luvas, mas não trabalham com procedimentos que envolvam esparadrapos; que realizam funções administrativas e que apresentassem alergia ao látex.

$\mathrm{O}$ protocolo adotado para coleta de dados seguiu duas etapas. A primeira diz respeito à realização de procedimentos que envolvam o uso concomitante de luvas e esparadrapos sem o 
uso do gel, seguida de um questionário na qual os profissionais destacavam as dificuldades apresentadas ao longo da assistência.

A segunda etapa adotada consistiu na demonstração do gel e instruções de como este deveria ser utilizado, seguido de distribuição de amostras para os participantes usarem durante a execução de procedimentos que envolvessem o uso de luvas e esparadrapos, sendo convidados posteriormente a preencher um questionário de satisfação, respondendo a seguinte pergunta: "Com a utilização do produto, que problemas foram sanados durante o uso de luvas nos procedimentos com esparadrapos?”.

O gel adotado neste estudo trata-se do gel condutor utilizado para a realização de eletrocardiograma, pois possui ph neutro, inodoro e não danifica o látex da luva e nem traz nenhum malefício a pele.

As instruções quanto ao método adequado de utilização do produto para que este fosse eficaz consistiu em após calçar as luvas, os participantes passem 5 mililitros do produto sobre as mãos já enluvadas, em toda a sua extensão (dorso e palma), sendo deixada uma quantidade extra, sem espalhar no dorso. Os participantes foram orientados a iniciarem a manipulação do esparadrapo imediatamente após a aplicação do gel, pois pode haver evaporação do produto, diminuindo sua eficácia.

Os participantes foram instruídos também que caso retorne a aderência da luva ao esparadrapo, este recorra ao produto extra que se encontrava depositado no dorso das mãos, fazendo novamente a umectação dos dedos e retornando com a sua ação sobre os dedos que manuseiam o esparadrapo. Os profissionais também foram orientados que caso no dorso da mão não houvesse quantidade suficiente de produto para garantia da não aderência fosse realizada a reaplicação de 5 mililitros do produto com a mesma técnica anteriormente citada.

Foi recomendada a quantidade de 5 mililitros de gel para a realização do manuseio do esparadrapo, pois esta quantidade foi a que se mostrou mais eficaz durante testes previamente realizados durante o desenvolvimento do produto, o uso em menor ou maior quantidade não garantem a boa usabilidade do produto em relação a não aderência ao esparadrapo, podendo o uso em maior quantidade reduzir a capacidade adesiva do esparadrapo até que o produto tenha sua total secagem.

Para preservar o anonimato dos participantes, na apresentação dos resultados, os enfermeiros foram nomeados com a letra "E" e o número sequencial, sendo os dados referentes às perguntas de identificação das dificuldades vivenciadas e do questionário aplicado após a utilização do gel analisadas mediante a técnica de análise de conteúdo, com 
abordagem temática, proposta por Bardin, seguindo as etapas de pré-análise, descrição analítica e tratamento dos resultados (Urquiza \& Marques, 2016).

A análise dos dados fez emergirem quatro categorias temáticas, sendo estas: dificuldades vivenciadas durante a utilização de luvas em procedimentos que contenham utilização de esparadrapos; rompimento das luvas e o desperdício de material; dificuldades e comprometimento da assistência relacionada à associação luvas-esparadrapo; os benefícios do gel durante as ações assistenciais.

\section{Resultados e Discussão}

A análise das falas dos participantes do estudo permitiu identificar que a associação das luvas com esparadrapos durante procedimentos promovem repercussões negativas a assistência, sendo estas repercussões trazidas à tona por intermédio do relato dos participantes. Deste modo, os resultados obtidos foram analisados e as categorias temáticas identificadas são apresentadas a seguir:

\section{Categoria I: Dificuldades vivenciadas durante a utilização de luvas em procedimentos que contenham utilização de esparadrapos}

As dificuldades vivenciadas durante a utilização de luvas em procedimentos que contenham utilização de esparadrapos aparecem na fala dos participantes quando estes realizam seus relatos, estando esta dificuldade associada à cola presente no material fixador.

A dificuldade na hora de retirar o esparadrapo, pois ele cola na luva, dependendo do curativo, profissionais acabam retirando a luva para poder fixar. O esparadrapo com o manuseio da luva se cola e acaba sendo desperdiçado muito mais. E1

Esparadrapo agarrava na luva, dificultando o uso. E3

Dentre um dos principais problemas na utilização do esparadrapo é a dificuldade que temos com a cola do esparadrapo na luva (quanto mais você tenta tirá-lo mais gruda). E18 


\section{Categoria II: Rompimento das luvas e o desperdício de material}

A aderência entre as luvas e o esparadrapo citada pelos participantes é referenciada como a responsável pelo desperdício de material visto que ocorre o rompimento das luvas. Deste modo, os profissionais acabam apontando mesmo que de forma indireta que este fator onera em custos para os estabelecimentos de saúde.

O uso da luva nos procedimentos que utilizam o esparadrapo fica difícil o manuseio por grudarem e rasgarem as luvas fazendo com que descartem as luvas para manusear. E5

As luvas rasgavam, dificultava o trabalho de punção, atrapalhando na realização de curativos, assim de uma forma geral a realização do trabalho de enfermagem. E9

Aderência excessiva do esparadrapo na luva, ocasionando rompimento da integralidade da luva. E16

\section{Categoria III: Dificuldades e comprometimento da assistência relacionada à associação luvas-esparadrapo}

Outro ponto que merece destaque na fala dos participantes é a dificuldade na execução de procedimentos quando se associa as luvas com o esparadrapo, o que compromete a assistência e expondo os profissionais ao risco de contato com material biológico e consequentemente podem resultar em afastamentos dos profissionais de suas funções se estes sofrerem acidentes de trabalho.

O problema maior é o esparadrapo ficar aderindo à luva, causando assim uma dificuldade na execução do procedimento. E10

O esparadrapo adere à luva dificultando a fixação dos curativos e demais procedimentos. Muitas vezes a luva rasga e expondo-nos a secreções. E14 
Research, Society and Development, v. 9, n. 7, e173974042, 2020

(CC BY 4.0) | ISSN 2525-3409 | DOI: http://dx.doi.org/10.33448/rsd-v9i7.4042

A fixação do esparadrapo na luva torna quase impossível alguns procedimentos, como por exemplo, fixar SOG em RNs, manipular e fixar o curativo da punção venosa. E15

Dificuldade de realizar os procedimentos, pois o esparadrapo gruda na luva; O esparadrapo danifica a luva, ficando assim o profissional exposto podendo se contaminar; Risco de acidente com acesso periférico, profundo, dissecção venosa e outros procedimentos realizados pelo profissional, com o risco de retirar o cateter junto com o esparadrapo. E31

A demora na realização de procedimentos também foi associada à dificuldade de se manusear esparadrapos com luvas e este ponto pode trazer repercussões irreversíveis em situações emergenciais.

Esparadrapo e luva são incompatíveis. Temos grandes problemas nesse manuseio, na maioria das vezes a luva que estamos utilizando rasga, por conta desse esparadrapo e perdemos bom tempo nesses procedimentos. E19

O esparadrapo é um material muito utilizado na rede hospitalar e seu uso com a luva de procedimento não é tarefa fácil para os profissionais de saúde. O mesmo gruda e rasga as luvas, o esparadrapo embola e fica inutilizável. Isso acarreta demora e atraso nos procedimentos, além de maior gasto do produto. E22

Frente a este cenário onde se evidencia o comprometimento da assistência, da segurança dos profissionais e consequentemente do aumento dos gastos com insumos (luvas e esparadrapos) em decorrência da aderência e rompimento, o estudo propôs que os pesquisados fizessem o uso do gel durante os procedimentos e os relatos evidenciaram que houve redução das dificuldades durante o uso de luvas nos procedimentos com esparadrapos. 


\section{Categoria IV: Os benefícios do gel durante as ações assistenciais}

Os profissionais apontaram em seus relatos após o uso do gel, o que acharam da utilização do produto e os relatos demonstram os aspectos positivos do produto dentre estes redução da aderência entre as luvas e esparadrapo que eram apontadas como um problema.

O produto é ótimo, evita que a luva fique grudando. E2

Esparadrapo não agarra na luva, facilitando todo o processo de trabalho. E3

Com a utilização do produto, a aderência ao esparadrapo se tornou mínima, facilitando assim um bom trabalho. E4

Realmente foi muito útil à utilização do gel condutor para a manipulação da luva com esparadrapo. E8

A redução na aderência também foi evidenciada como um ponto positivo no que se refere a evitar o desperdício de materiais por profissionais que rompem as luvas durante as ações, bem como apresenta ação positiva frente a evitar o risco de exposição do profissional a materiais biológicos, seja por rompimento da luva ou por retirar a luva durante a assistência com vistas à fixação do esparadrapo.

Não houve perda do esparadrapo bem como rasgos na luva. O esparadrapo não perde sua cola, não havendo assim desperdício do material. E11

$O$ uso do gel não deixa grudar o esparadrapo na luva facilitando o manuseio e não permitindo que o profissional faça o descarte das luvas para o manuseio do esparadrapo permitindo que o mesmo possa vir a se contaminar. E5

Não houve aderência do esparadrapo na luva facilitando a utilização do mesmo sem risco de contaminação do profissional em caso de sujidade com material biológico. E6 
Research, Society and Development, v. 9, n. 7, e173974042, 2020

(CC BY 4.0) | ISSN 2525-3409 | DOI: http://dx.doi.org/10.33448/rsd-v9i7.4042

Com a utilização deste produto não é mais necessário retirar as luvas para fixar o esparadrapo e nem tendo a possível contaminação com fluidos como sangue, secreção. E20

Os participantes também apontam que o uso do gel facilita o trabalho o que consequentemente pode vir a contribuir para a redução do tempo que os profissionais perdem na incessante luta "luvas-esparadrapos".

O esparadrapo não cola mais na luva, porém não perdia a cola para uso de tal função, facilitando o trabalho da enfermagem. E9

O produto não deixa colar na luva e não perde a cola para colar na pele. E12

O produto evitou a aderência do esparadrapo à luva e manteve a eficácia na cola do produto em outras superfícies sem ser a luva. E14

A luva não grudou no esparadrapo, contribuindo para o sucesso do procedimento realizado. E15

Não teve aderência excessiva do esparadrapo com luva, podendo realizar corte do esparadrapo e aplicação sem dificuldades. E16

Facilidade ao tentar desgrudar o esparadrapo da luva, agilidade no manuseio. E17

Primeiro não grudar na luva; Segundo não correr o risco de puxar o curativo causando lesão no paciente; Terceiro quando se usa o produto causa uma refrescancia na mão o que dá conforto ao profissional. E18

Frente o exposto, torna-se fundamental as discussões acerca da usabilidade do gel para evitar a aderência do esparadrapo a luvas visto que estes quando usados sem o uso da tecnologia (gel) comprometem a assistência e a segurança do paciente e do profissional de saúde. 
O uso de luvas é imprescindível na prevenção da infecção associada à assistência, devendo a sua utilização ser adotada sempre que houver a realização de procedimentos, devendo a mesma ser retirada após a finalização (Silva, et.al., 2016), mas o que observa-se na prática é a retirada do EPI previamente quando se deseja manusear esparadrapos. Esta postura expõe os profissionais de enfermagem a riscos de contato com fluidos corporais dos pacientes, bem como também leva o paciente a ter contato com microrganismos presentes nas mãos dos profissionais.

Nas instituições de saúde, a assistência de enfermagem é fundamental para melhoria da segurança do paciente (Silva, et.al., 2016), porém evidenciamos em muitos casos condutas de imprudência e negligencia relacionada a retirada das luvas quando pretende-se fixar o esparadrapo.

Os resultados do estudo apontam que os profissionais sabem dos riscos que a não utilização das luvas ou o rompimento das mesmas podem ocasionar, ou seja, os pesquisados sabem que a utilização do EPI e o emprego de práticas seguras contribuem significativamente para a redução do risco de acidentes ocupacionais e infecções (Corrêa, et.al., 2017).

Nesta perspectiva, evidencia-se que a adoção de tecnologias que venham a corroborar com a prática assistencial e consequentemente promover a segurança dos pacientes e dos profissionais de saúde torna-se primordial (Balbino, et.al., 2020). Logo, o uso do gel associado à luva de látex fornece repercussões positivas à assistência.

O uso do gel associado à luva de látex fornece repercussões positivas à assistência, pois possibilita que o manuseio do esparadrapo seja compatível, evitando que a mesma seja danificada ou retirada na hora da realização da técnica melhorando a assistência de enfermagem prestada ao indivíduo com a diminuição dos riscos de contaminação por parte do profissional, visto que ao serem aderidos, na maioria das vezes são descartados, tornando-os sem utilidade, pois com o dano ao material (luva ou esparadrapo) há inviabilidade do seu uso. Destarte, o uso da tecnologia em questão (gel), resulta em economia de material, evitando o desperdício observado nos hospitais (Nascimento, 2015).

Torna-se importante destacar que para o desenvolvimento de tecnologias a favor da melhor assistência é necessário estar realizando ações assistenciais e de cuidado ao paciente, visto que a possibilidade de se criar novas tecnologias só pode ser vislumbrada durante a prática (Nascimento, 2015). Deste modo, o enfermeiro é um ator primordial na identificação destas demandas, por ele estar cuidando constantemente do paciente. Ou seja, o referido profissional consegue vislumbrar as demandas tecnológicas necessárias a uma assistência segura e de qualidade. 
Frente o exposto, os resultados evidenciados neste estudo e apresentados acima demonstram que a tecnologia pode ser usada com outras finalidades em benefício da assistência segura como o gel condutor utilizado comumente na realização de eletrocardiogramas que foi vislumbrado por enfermeiros como um importante aliado tecnológico na prática assistencial, devendo para esta adoção os profissionais serem orientados sobre como proceder, sendo importante neste caso a capacitação e treinamentos específicos sobre a importância do EPI e da sua utilização adequada, além de orientações quanto as demais normas de biossegurança (Corrêa, et.al., 2017).

Uma das limitações a ser considerada no desenvolvimento do estudo diz respeito aos achados serem caracterizados mediante a investigação em apenas um cenário, o que pode caracterizar a homogeneidade no perfil dos pesquisados e nas vivencias experenciadas por eles.

Outra limitação encontrada diz respeito a artigos em bases nacionais e internacionais que viessem a contribuir para a discussão acerca da usabilidade da tecnologia do gel para utilização em luvas para melhor manuseio com esparadrapos. Esta limitação ressalta a importância de serem desenvolvidas novas pesquisas que prezem pela adoção de tecnologias com vistas ao não comprometimento da assistência quando associadas ao uso do EPI, proporcionando maior acervo aos pesquisadores, profissionais e estudantes da área da saúde, tendo em vista o fato das luvas serem utilizadas constantemente durante as práticas assistenciais.

\section{Considerações Finais}

Os resultados presentes neste estudo apontam que o uso do gel como tecnologia para evitar a aderência entre as luvas e o esparadrapo em decorrência da cola é positivo e apresenta usabilidade que tende a favorecer a assistência não apenas dos profissionais de enfermagem, mas dos diversos profissionais de saúde.

Os benefícios são estendidos a todos os profissionais de saúde que se utiliza de luvas e que tenham contato com materiais biológicos, visto que os achados apontam que a tecnologia estudada impede o rompimento do EPI quando associado à cola do esparadrapo.

Como a tecnologia do gel já existe, por tratar-se do gel condutor para a realização de eletrocardiogramas, e apenas teve seu uso redirecionado, evidenciamos que a partir da prática assistencial de enfermagem, é possível determinar a criação e/ou adaptações de tecnologias, para resolver os problemas visualizados na assistência em saúde. 
Os achados promovem contribuições para a assistência em saúde por promover a redução da aderência entre as luvas e o esparadrapo evitado que o profissional tenha dificuldades em suas ações e retire as luvas para fixar o esparadrapo o que consequentemente expõe este ao contato com materiais biológicos. Outra contribuição do estudo diz respeito à redução de luvas rompidas pela aderência da cola do esparadrapo no material de látex que compõe o referido EPI

Destarte, evidenciam-se por intermédio dos resultados que além da biossegurança, são contemplados aspectos relativos à química (gel), administração hospitalar (economia hospitalar), microbiologia (contaminação por contato), entre outros.

Os achados também demonstram que o uso do gel influencia na dinâmica assistencial, promovendo a segurança dos profissionais que não abandonam o uso do EPI para realizar atividade de fixação e consequentemente ocorre à redução da exposição deste aos fluídos, promove a continuidade das ações assistenciais sem pausas desnecessárias para trocas de luvas por rompimento ou aderência o que pode contribuir para a redução do tempo gastos nos procedimentos.

Deste modo, sugere-se que estudos realizados com a associação luvas-gel-esparadrapo sejam realizados com vistas a mensurar se há de fato redução de luvas desperdiçadas e consequentemente redução de custos nas unidades de saúde no que se refere à compra do EPI e redução de tempo inerente a ações assistenciais.

\section{Referências}

Balbino, C.M, Silvino, Z.R., Joaquim, F.L., Souza, C.J. \& Santos, L.M.(2020). Inovação tecnológica: perspectiva dialógica sob a ótica de Joseph Schumpeter. Research, Society and Development, 9 (6), e198963593.

Barboza, M.C.N., Almeida, M.S., Rodeghiero, J.B.H, Louro, V.A., Bernardes, L.S. \& Rocha, I.C.(2016). Riscos biológico e adesão a equipamentos de proteção individual: percepção da equipe de enfermagem hospitalar. Rev Pesq Saúde, 17(2),87-91.

Barros, J.S.O., Rodrigues, A.P.R.A., Miranda, L.N. \& Araujo, M.A.S. (2016). Nursing and resistance to the use of equipments for individual safety. Cad Grad Ciênc Biol Saud Unit, 3(3), 189-200. 
Brasil. (2006). Ministério do Trabalho e Emprego (BR). Portaria $n^{\circ} 194$ de 22 de dezembro de 2006. NR6 - Equipamentos de Proteção Individual. Recuperado em 10 de outubro de 2019, http:// www.mte.gov.br/legislacao/normas_regulamentadoras/nr_06.pdf

Brasil. (2008). Agência Nacional de Vigilância Sanitária (BR). Resolução da diretoria colegiada $n^{\circ} 05$ de 15 de fevereiro de 2008. Estabelece os requisitos mínimos de identidade e qualidade para as luvas cirúrgicas e luvas de procedimentos não-cirúrgicos de borracha natural, borracha sintética ou mistura de borrachas natural e sintética, sob regime de vigilância sanitária. Recuperado em 30 de novembro de 2019, http://www.sbpc.org.br/upload/conteudo/rdc5_2008.pdf

Brasil. (2018). Agência Nacional de Vigilância Sanitária (BR). O primeiro desafio mundial para a segurança do paciente: uma assistência limpa é uma assistência mais segura - uso de luvas (técnico). Recuperado em 13 de outubro de 2019, http://www.anvisa.gov.br/servicosaude/controle/higienizacao_oms/folha\%20informativa\%20 6.pdf

Corrêa, L.B.D., Gomes, S.C.S., Ferreira, T.F. \& Caldas, A.J.M. (2017). Factors associated with use of personal protective equipment by healthcare professionals who suffered accidents with biological materials in the State of Maranhão. Rev Bras Med Trab, 15(4):340-9.

International Organization for Standardization (ISO).(2018) Ergonomics of human-system interaction -Part 11: Usability: Definitions and concepts, 2: 1-29 Recuperado em 10 de novembro de 2019, http://www.iso.org/standard/63500.html

Meneses, F.A.V., Sobreira, E.J. \& Rosemberg, D.S. (2016) Estado do conhecimento sobre usabilidade na revista Ciência da Informação. Ciência da Informação, 45(1), 193-4.

Nascimento, M.A.L. (2005). The adhesive plasterer: adaptation of a technology in nursing care. Rev Enferm UERJ, 13 (1), 63-7.

Silva, A.T., Alves, M.G., Sanches, R.S., Terra, F.S. \& Resck, Z.M.R. (2016) Nursing care and the focus on patient safety in the Brazilian scenario. Saúde Debate, 40 (111), 292-301. 
Sulzbacher, E. \& Fontana, R.T.(2018). Conceptions of nursing staff about the exposure to physical and chemical risks in hospital environment. Rev Bras Enferm, 66 (1), 25-30.

Urquiza, M.A. \& Marques, D.B. Análise de conteúdo em termos de Bardin aplicada à comunicação corporativa sob o signo de uma abordagem teórico-empírica. Entretextos, 16 (1), 115-44.

Vieira, N.A., Lima, D.W.C., Silva, F.T. \& Oliveira, G.W.S. (2015). Use of personal protective equipment for nursing professionals in primary health care. Rev Enferm UFPE On Line, 9(10),1376-83.

\title{
Porcentagem de contribuição de cada autor no manuscrito
}

\author{
Carlos Marcelo Balbino - 40\% \\ Zenith Rosa Silvino - 10\% \\ Fabiana Lopes Joaquim - 20\% \\ Cláudio José de Souza - 10\% \\ Ana Paula Bergmann Furtado Costa - 10\% \\ Lívia Luiz de Fátima Moura - 10\%
}

